\title{
Postoperative Chemotherapy for Thoracic Pathological T3N0M0 Esophageal Squamous Cell Carcinoma
}

\author{
Audrey E. Kam, MD', Sam G. Pappas, $\mathrm{MD}^{2}$, and Ashiq Masood, MD ${ }^{1}$ \\ ${ }^{1}$ Division of Hematology, Oncology and Cell Therapy, Rush University Medical Center, Chicago, IL; ${ }^{2}$ Division of Surgical \\ Oncology, Rush University Medical Center, Chicago, IL
}

Esophageal squamous cell carcinoma (ESCC) remains the major cause of cancer-related mortality in China. Recent molecular studies have shown a different mutational profile in ESCC patients from China compared with the Western population, owing to different mutagen exposure and highlighting the heterogeneous nature of ESCC globally. ${ }^{1}$ Therefore, a 'one-size-fits-all' strategy may not be applicable across different geographic regions. Consequently, the standard of care for locally advanced ESCC varies between the West and Asia, including China.

Surgery continues to be the critical component in the management of locally advanced ESCC (T3-4aN0, T1-4aN1); however, the 5-year overall survival (OS) rate with surgery alone remains low $(15-24 \%) .^{2}$ In the West, neoadjuvant chemoradiotherapy (NACRT) plus surgery has become standard practice in ESCC, primarily based on the CROSS trial, albeit only $23 \%$ of patients having squamous cell histology. ${ }^{3}$ In Japan, two large studies (JCOG 9204 and JCOG 9907) have led neoadjuvant chemotherapy (NAC) plus surgery to become the standard treatment strategy. ${ }^{4,5}$ In China, the phase III trial 'Neoadjuvant Chemoradiotherapy Followed by Surgery Versus Surgery Alone for Locally Advanced Squamous Cell Carcinoma of the Esophagus (NEOCRTEC5010)', showed disease-free survival (DFS) and OS benefit with NACRT in T1-4N1 and T4N0 ESCC. ${ }^{6}$ It is worth noting that the

(C) Society of Surgical Oncology 2019

First Received: 25 November 2019; Published Online: 17 December 2019

A. E. Kam, MD

e-mail: Audrey_E_Kam@rush.edu majority of patients in NEOCRTEC5010 had N1 disease. Nevertheless, the study demonstrated a significant OS benefit with the incorporation of NACRT to surgery in a Chinese patient population.

The therapeutic benefit of adjuvant therapy in esophageal cancer is an ongoing debate due to the lack of highquality randomized data. Several retrospective studies have evaluated the role of adjuvant chemotherapy (AC) in locally advanced ESCC, with conflicting results in terms of DFS and OS. ${ }^{7-9}$ In a large meta-analysis of 11 studies with over 2000 patients, the DFS benefit of AC was only seen in lymph node (LN)-positive patients, and OS benefit was limited to stage III/IV disease. ${ }^{10}$ With regard to adjuvant chemoradiotherapy (ACRT), retrospective studies have shown a survival benefit in LN-positive disease, ${ }^{11,12}$ while one study found a survival benefit in T3NOM0 patients. ${ }^{13}$ Thus, current Chinese guidelines for ESCC recommend surgery alone for resected (R0) pT2-3NOM0; neoadjuvant therapy in stage III (T3N1M0-T4N0-1M0); and adjuvant therapy for pT4N0 or T1-4N1-3M0. ${ }^{14}$

In the current study by Wang et al., ${ }^{15}$ the authors attempt to clarify the role of adjuvant therapy in PT3N0 thoracic ESCC patients who underwent upfront surgical resection. The 5-year DFS and OS rates were significantly improved in patients treated with surgery plus postoperative chemotherapy (S+POCT) compared with surgery alone (S) (DFS: $66.5 \%$ vs. $50.2 \%, p=0.002$; OS: $70.8 \%$ vs. $52.8 \%, p<0.0001)$. Furthermore, on subgroup analysis, both DFS and OS benefit were maintained in patients with $\leq 20$ LNs removed or pStage IIB, but not in those with $>20$ LNs removed or pStage IIA.

This timely publication explicitly addresses a subset of locally advanced ESCC patients with LN-negative disease, while the majority of the aforementioned $\mathrm{AC}$ trials focused 
on LN-positive patient populations. To add to its strength, the study was fairly sizeable, with over 580 patients, and utilized propensity score matching to balance comparison groups.

While the results of this study are thought-provoking, some limitations are noteworthy. First, the complication rate in the $\mathrm{S}$ group was increased significantly compared with the S+POCT group (71/236 [30.1\%] vs. 50/236 [21.2\%]; $p=0.027$ ), which may have led to selection bias for patients with better prognosis as well as patients who could tolerate systemic treatment in the S+POCT cohort. Second, the chemotherapy regimen was highly variable, including the number of cycles administered (median 3; range 1-6). Third, the DFS and OS benefit of POCT was only maintained in patients with $\leq 20$ LNs removed or pStage IIB, limiting its generalizability. In the case of patients with $<20$ LNs removed, POCT may make up for inadequate LN dissection, which was also reflected by a decrease in the locoregional relapse rate $(16.5 \%$ vs. $25.0 \%$; $p=0.023$ ), but not in the rate of distant metastases with POCT. Lastly, the results are difficult to generalize as this was a retrospective, single-institution study. Furthermore, its applicability on a global level is limited since the treatment paradigm in Japan and Western countries is primarily centered on neoadjuvant therapies. Therefore, evidence for adjuvant therapy for T3NO ESCC is not sufficient to warrant changes to the current Chinese guidelines.

To conclude, Wang et al. demonstrate an improvement in DFS and OS with POCT in upfront resected (R0) pT3NOM0 thoracic ESCC. ${ }^{15}$ The study is hypothesis-generating and suggests that patients with locally advanced LN-negative ESCC may benefit from adjuvant therapy. Obviously, prospective studies are needed to confirm these findings before adopting adjuvant therapy strategies in T3NO ESCC into clinical practice. The ongoing, prospective, phase III NCT02891083 trial in China is currently underway to evaluate the role of adjuvant therapy (ACRT or AC) versus surgery alone in resected pT1b-T4aN0 ESCC with high-risk features, i.e. upper/middle thoracic lesion, lymphovascular invasion, submucosal metastasis, or poor differentiation. Furthermore, several phase III trials are being conducted to assess the role of neoadjuvant therapy in locally advanced ESCC (NCT03001596, NCT02395705), which may potentially shift the treatment paradigm. As more high-quality prospective data from the aforementioned studies become available, clinicians will be able to better define the best approach for the treatment of locally advanced ESCC patients.

DISCLOSURES Audrey E. Kam, Sam G. Pappas, and Ashiq Masood declare no conflicts of interest.

\section{REFERENCES}

1. Deng J, Chen H, Zhou D, et al. Comparative genomic analysis of esophageal squamous cell carcinoma between Asian and Caucasian patient populations. Nat Commun. 2017;8(1):1533.

2. Enziger PC, Mayer RJ. Esophageal cancer. $N$ Eng J Med. 2003;349:2241-52.

3. van Hagen P, Hulshof MCCM, van Lanschot JJB, et al. Preoperative chemoradiotherapy for esophageal or junctional cancer. $N$ Engl J Med. 2012;366:2074-84.

4. Ando N, Iizuka $\mathrm{T}$, Ide $\mathrm{H}$, et al. Surgery plus chemotherapy compared with surgery alone for localized squamous cell carcinoma of the thoracic esophagus: a Japan Clinical Oncology Group Study-JCOG9204. J Clin Oncol. 2003;21:4592-6.

5. Ando N, Kato H, Igaki $\mathrm{H}$, et al. A randomized trial comparing postoperative adjuvant chemotherapy with cisplatin and 5-fluorouracil versus preoperative chemotherapy for localizedadvanced squamous cell carcinoma of the thoracic esophagus (JCOG9907). Ann Surg Oncol. 2012;19:68-74.

6. Yang H, Liu H, Chen Y, et al. Neoadjuvant chemoradiotherapy followed by surgery versus surgery alone for locally advanced squamous cell carcinoma of the esophagus (NEOCRTEC5010): a phase III multicenter, randomized, open-label clinical trial. J Clin Oncol. 2018;36(27):2796-803.

7. Lee J, Lee KE, Im YH, et al. Adjuvant chemotherapy with 5-fluorouracil and cisplatin in lymph node-positive thoracic esophageal squamous cell carcinoma. Ann Thorac Surg. 2005;80:1170-5.

8. Qin RQ, Wen YS, Wang W, et al. The role of postoperative adjuvant chemotherapy for lymph node-positive esophageal squamous cell carcinoma: a propensity score matching analysis. Med Oncol. 2016;33:31.

9. Lyu X, Huang J, Mao Y, et al. Adjuvant chemotherapy after esophagectomy: Is there a role in the treatment of the lymph node positive thoracic esophageal squamous cell carcinoma? J Surg Oncol 2014;110:864-8.

10. Zhang SS, Yang H, Xie X, et al. Adjuvant chemotherapy versus surgery alone for esophageal squamous cell carcinoma: a metaanalysis of randomized controlled trials and nonrandomized studies. Dis. Esophagus 2014;27(6):574-84.

11. Chen J, Pan J, Liu J, et al. Postoperative radiation therapy with or without concurrent chemotherapy for node-positive thoracic esophageal squamous cell carcinoma. Int J Radiat Oncol Biol Phys. 2013;86(4):671-7.

12. Hsu PK, Huang CS, Wang BY, et al. Survival benefits of postoperative chemoradiation for lymph node-positive esophageal squamous cell carcinoma. Ann Thorac Surg. 2014;97(5):1734-41.

13. Yang J, Zhang W, Xiao Z, et al. The impact of postoperative conformal radiotherapy after radical surgery on survival and recurrence in pathologic T3NOM0 esophageal carcinoma: a propensity score-matched analysis. $J$ Thorac Oncol. 2017;12:1143-51.

14. National Health Commission of the People's Republic of China. Chinese guidelines for diagnosis and treatment of esophageal carcinoma 2018 (English version). Chin J Cancer Res. 2019;31(2):223-58.

15. Wang Q, Peng L, Li T, et al. Postoperative chemotherapy for thoracic pathological T3NOM0 esophageal squamous cell carcinoma. Ann Surg Oncol. 2019. https://doi.org/10.1245/s10434019-08112-1.

Publisher's Note Springer Nature remains neutral with regard to jurisdictional claims in published maps and institutional affiliations. 